\title{
Alfabetización informacional, innovación evaluación como funciones de la biblioteca universitaria del siglo XXI: visión desde un enfoque cualitativo
}

Information literacy, innovation and assessment as essential functions of the twenty-first century academic library: a view from a qualitative perspective

María Pinto Molina (1), María de las Mercedes Fernández ValdÉs (2)

(1) Universidad de Granada, mpinto@ugr.es (2) Centro Nacional de Información de Ciencias Médicas-Infomed, mercy@infomed.sld.cu

\section{Resumen}

Las bibliotecas universitarias se enfrentan a los cambios cualitativos impuestos por la sociedad de la información y el conocimiento en los procesos, productos y servicios. Incorporar al flujo de trabajo de la biblioteca aspectos como la alfabetización informacional, la evaluación y la innovación constituye un reto esencial para la universidad actual y del futuro. A partir de un focus group realizado por profesionales de la información de América Latina y el Caribe, se describen cómo estos conceptos van integrándose en la filosofía de trabajo de estas instituciones y de las nuevas competencias que los bibliotecarios y documentalistas deben dominar para lograr el avance de la profesión hacia nuevos horizontes.

Palabras claves: Bibliotecas universitarias Alfabetización informacional. Innovación. Evaluación de la calidad.

\section{Introducción}

El tránsito de la "sociedad industrial" a la "sociedad de la información y el conocimiento" constituye razón suficiente para que a nivel global las organizaciones hayan tenido que suplir los estilos cerrados y conservadores de trabajo por una filosofía abierta a los cambios y orientada al aprendizaje de las personas y de la propia organización. Aunque dicho así puede parecer sencillo, son muchas las organizaciones que hoy buscan estrategias para lograr acomodarse a las exigencias de un mundo donde de manera constante se renueva la información y los profesionales de todas las ramas del saber han de estar preparados para enfrentarse a la difícil tarea de aprender de manera continua nuevos procederes y técnicas relacionadas con su especialidad.

El éxito de estas personas que han sido formadas para desarrollar y aplicar el conocimiento

\begin{abstract}
:
Academic libraries are facing the changes imposed by the information and knowledge society, and, in particular, the need to achieve a consistent qualitative change in their processes and services. Incorporating to the traditional flow of library work areas such as information literacy, evaluation and innovation is an essential challenge for present and future universities. This paper describes how these concepts are beginning to join the working philosophy of these institutions and how the new competencies that librarians and archivists must learn to achieve the advancement of the profession to new horizons are being incorporated. It is based in the results and conclusions from a focus group conducted by professionals from Latin America and the Caribbean in the field of library and information science.
\end{abstract}

Keywords: Academic libraries. Information literacy. Innovation. Quality assessment.

depende en gran medida del espacio donde estudiaron: la universidad, piedra angular para garantizar la formación en el presente y en el futuro de individuos preparados para analizar los fenómenos desde una perspectiva abierta al cambio, que involucre lo simple y lo complejo, lo disciplinar y lo transdisciplinario, lo nuevo y lo desconocido, lo relacionado con la ciencia, la ética, el desarrollo personal y la justicia social. En este enclave, la biblioteca considerada desde la legendaria "Alejandría" símbolo del saber, la inteligencia y el conocimiento, juega un papel vital que ha de re-actualizar y redefinir. Sirva de reflexión la siguiente frase acuñada en 1976 en el conocido Informe Atkinson, donde se afirmaba que (University Grants Commitee, 1976).

la biblioteca es el corazón de la universidad, ocupando un lugar central y básico, como un recurso que es, ocupa y sirve a todas las funciones de una universidad: enseñanza e investigación, creación 
de nuevo conocimiento y la transmisión a la posteridad de la ciencia y la cultura del presente.

\section{Revisión de la literatura}

Diversos son los autores contemporáneos que abordan la problemática de la biblioteca universitaria desde una perspectiva de cambio, flexibilidad y dinamismo, rompiendo con los muros que durante siglos rodeo todo lo relacionado con la formación y los servicios universitarios. Plantean el tránsito hacia una biblioteca conceptualmente diferente que debe contar con una estructura funcional dinámica que le permita gestionar recursos y servicios de información independientemente del soporte o la localización que tengan y proporcionar el acceso a esos recursos a través de diferentes medios y en diferentes puntos (Pinto, Sales y Osorio, 2008).

Los cambios que se producen en la biblioteca universitaria son directamente proporcionales a los que ocurren en la propia universidad. La universidad debe dotar a la biblioteca de infraestructuras y equipamientos que permitan la innovación metodológica, formar e integrar las TICs en los nuevos modelos de enseñanzaaprendizaje. Impulsar la colaboración de expertos en tecnologías, profesores y bibliotecarios, con la finalidad de producir y organizar contenidos y recursos docentes y participar en proyectos transversales: publicación electrónica de trabajos científicos, tesis, dossieres, repositorios, etc. (Domínguez Aroca, 2005)

En el contexto de la actual sociedad del conocimiento y del aprendizaje la biblioteca universitaria es definida como una organización sistémica, compleja, flexible y con capacidad de adaptarse a los nuevos modelos de enseñanza/aprendizaje, más versátiles y personalizados, centrados en la transferencia de habilidades y competencias informacionales para la toma de decisiones y para el uso, aprovechamiento y difusión de la información. (Pinto, Balagué y Anglada, 2007).

La biblioteca universitaria apoya la docencia, el aprendizaje y la investigación, y se convierte en el eje esencial para el desarrollo de los procesos de gestión de información y gestión del conocimiento que soportan estas actividades, sin las cuales la universidad dejaría de existir. Como señala Sánchez Vignau (2007) queda demostrado que para cumplir con la política científica de la universidad, el uso y el manejo de la información resulta un aspecto necesario e importante. Esto hace que la universidad exija a su biblioteca:
- Una garantía de colecciones con contenidos de alto valor científico.

- Servicios de seguimiento de los resultados de la investigación.

- El desarrollo de sistemas editoriales que muestren el quehacer investigador y científico.

- El control de perfiles de interés de las comunidades científicas universitarias.

- La atención a programas de formación a lo largo de la vida.

La biblioteca universitaria se convierte en el centro de acción para que los estudiantes desarrollen su aprendizaje y para que gestionen la información y el conocimiento en su formación como profesionales. El cambio es algo evidente, sobre todo si se hace referencia al paradigma de la alfabetización informacional como una forma más social, integradora y humana de que los estudiantes, comprendan la importancia de utilizar la información de modo ético y consciente, en todas las acciones que se realizan tanto en el ámbito personal, académico como profesional.

Pero los profesores también se encuentran en un momento diferente de desarrollo y uso de la biblioteca. El profesor ha de prepararse y adquirir conocimientos actualizados que le permitan la conducción del proceso de enseñanzaaprendizaje y la orientación adecuada a sus estudiantes en un entorno donde incluso el aprendizaje utilizando los entornos virtuales es algo novedoso y que debe ser objeto de estudio. Los docentes además de utilizar la biblioteca principalmente, como soporte de su docencia y medio básico de su actividad investigadora, habrán ahora de considerarla desde una perspectiva más amplia, pues serán espacios fundamentales para la generación de materiales orientados a la formación y mejora de competencias básicas y específicas, en distintos soportes, que formen parte de la programación ordinaria de las asignaturas de los nuevos planes de estudio (Moscoso, 2003).

La Universidad y su biblioteca tienen que prepararse para dar respuesta a las necesidades que surgen en consonancia con los cambios operados en la educación y formación de los individuos en la actual sociedad de la información y el conocimiento. La milenaria institución, esencial en la educación desde su surgimiento, se encuentra en un momento de tránsito hacia una universidad moderna, activa, flexible, profunda y transdisciplinaria.

Ante el uso intensivo de las TiCs en los procesos de aprendizaje, la balanza se inclina hacia la utilización combinada del aprendizaje presen- 
cial con las diferentes variantes de aprendizaje virtual, como el aprendizaje mixto o blended learning (Carman, 2002; Oyston, 2003; Garrison y Kanuka, 2004).

El cambio realizado en la biblioteca universitaria contribuye a la educación informacional de la comunidad universitaria, especialmente de los estudiantes (Kazmer, 2002). La nueva concepción de los servicios de la biblioteca tiene como misión ayudar a profesores y estudiantes en las actividades de aprendizaje, formación, gestión y resolución de problemas técnicos, metodológicos y de conocimiento en el acceso y uso de la información (Area, 2004).

La biblioteca universitaria, como se aprecia en el gráfico, se convierte en la piedra angular para:

- Ser soporte del aprendizaje.

- Liderar los procesos de alfabetización informacional y desarrollo de competencias informacionales que se requieran en la universidad.

- Educar a partir del precepto del aprender a aprender

- Impulsar todos los procesos relacionados con la innovación, el desarrollo y la calidad dentro de la universidad.

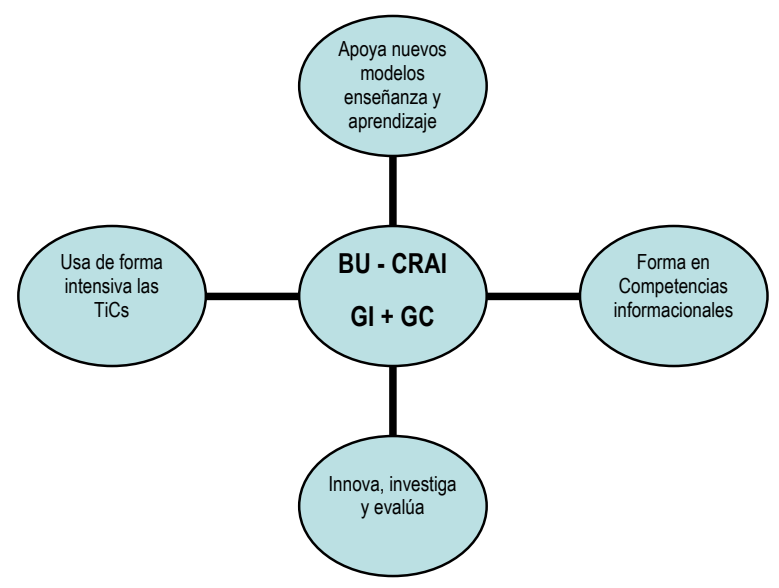

Figura 1

Esta nueva dimensión de las bibliotecas universitarias que va más allá de su función tradicional y la sitúa en el centro del desarrollo de los procesos de investigación y aprendizaje a partir de un nuevo modelo de organización y funcionamiento, tiene su génesis en los Learning Resources Centres (LRC), modelo anglosajón que define a un sistema de información altamente eficaz para localizar cualquier dato o recurso de información en el entorno académico de la uni- versidad y cuyo uso e implementación fueron planteados ya por Bultlar y Tipton (1992). Es valido hacer referencia además al Modelo Information Commons (Beagle, 1999), que plantea la combinación del espacio físico y virtual y la convergencia de los servicios de biblioteca, docencia e investigación.

Estos modelos orientados al Centro de Recursos para el Aprendizaje y la Investigación (CRAI) deben convertirse en el espacio para que los estudiantes desarrollen sus trabajos académicos y los profesores elaboren los materiales para el proceso formativo de los estudiantes. Además, suponen una respuesta estratégica para el apoyo y mejora de la calidad del aprendizaje del estudiante, ofreciendo una visión más amplia e integral de dicho apoyo, al hacer converger con los servicios de biblioteca otros de enorme utilidad tales como el servicio de informática, de idiomas, de recursos educativos y multimedia. (Pinto, 2008)

Por tanto, los CRAls deben ser un referente importante para la educación informacional de profesores y estudiantes, convirtiéndose en servicios facilitadores para el desarrollo de habilidades y competencias transversales relacionadas con aprender a aprender, saber localizar, evaluar y sintetizar información para la resolución de problemas y aprender a trabajar de manera autónoma (...) y preservando para el futuro la base de la información de la organización, garantizando su integridad y seguridad; y generar un clima de innovación y creatividad continuo de la institución mediante el uso de los recursos adecuados de información.

Así pues, la biblioteca plenamente integrada en el escenario académico de la institución constituye uno de los principales pilares para el aprendizaje autónomo, permitiendo acceder a las diversas fuentes de información, analizar críticamente la información obtenida y transformarla en conocimiento. Se han de emplear estrategias formativas holísticas, en colaboración con el equipo académico y vinculado al aprendizaje global de los estudiantes. La biblioteca ha de ser un centro para el aprendizaje donde compartir información y conocimiento.

De esta manera, la biblioteca universitaria/CRAI debe combinar entre sus funciones, actividades y roles, las siguientes:

1. Integrar en colaboración con los profesores, programas de alfabetización informacional que desarrollen en los estudiantes competencias para el acceso, localización y uso adecuado de la información. 
2. Jugar un rol fundamental en la motivación del estudiante en la búsqueda del nuevo conocimiento.

3. Brindar, además de los servicios tradicionales (referencia, sala de lectura, localización de bibliografía, etc.), servicios virtuales utilizando las bondades de las tecnologías de la Información y la comunicación.

4. El personal debe estar preparado para asumir los retos que le impone a la profesión todos estos nuevos cambios.

5. Las colecciones deben responder tanto a los intereses docentes, de aprendizaje como investigadores de la universidad.

6. Ofrecer el espacio, los medios y el tiempo para el desarrollo de la educación fuera de medios formales, convirtiéndose en parte activa del proceso de aprendizaje del estudiante.

7. Brindar servicios adaptados a las necesidades de información de todos los miembros y agentes de la comunidad universitaria, incluyendo la accesibilidad a las personas con discapacidad.

8. Añadir a sus procesos de trabajo la innovación y la creatividad, lo cual le permitirá incorporar a los servicios elementos que facilitarán la satisfacción de las necesidades de los usuarios.

Recientemente Harry Bruce (2009), decano de la Facultad de Información de la Universidad de Seattle esbozó algunas recomendaciones para las bibliotecas y los bibliotecarios de las instituciones académicas con una mirada hasta el 2020. Resulta interesante como plantea el cambio a partir de que los estudiantes de pre y postgrado y los profesores e investigadores serán miembros de una nueva generación (nativos digitales) y por tanto tendrán nuevas necesidades de información, nuevos conocimientos y nuevas formas de comunicación. Para este cambio se ha de preparar la biblioteca universitaria del presente, que tendrá que anticiparse a estas necesidades y crear servicios diferentes. Afirma, que la biblioteca será el espacio para la investigación, la colaboración, el desarrollo científico y la innovación.

En este contexto, se plantea el interrogante de cómo deberían los bibliotecarios caminar hacia un modelo de profesional más competente, con un marcado posicionamiento en el contexto docente e investigador y una significativa presencia activa en los procesos de aprendizaje de los estudiantes. Son numerosas las incógnitas pero también abundantes los frentes de trabajo relacionados con la innovación.

En la literatura publicada sobre el tema se ha abordado con fuerza la necesidad de trazar políticas que marquen el camino para desarrollar la innovación en los servicios, la cual tiene particularidades que la diferencian de la innovación tecnológica (Cunningham, 2006; Howells, 2000). Innovar no es sólo hacer cosas distintas sino hacer cosas mejores. Innovar no es estar cambiando constantemente (por aquello de identificar innovación con cambio) sino introducir variaciones como resultado de procesos de evaluación y ajuste de lo que se estaba haciendo. La cuestión es introducir procesos innovadores que vayan asentando prácticas que supongan una mejora de la calidad de lo que se está haciendo (Zabalza, 2003).

El bibliotecario, en la universidad, se convierte en un gestor de información y conocimiento y debe ser visionario para poder predecir la demanda de futuros servicios y poder actuar de manera prospectiva en el desarrollo de nuevos servicios. (Cano, 1996). Tendrá que estar preparado para afrontar la gestión del cambio, comprendiendo los caminos y las barreras hacia la innovación y la reorganización, definiendo nuevos roles y nuevas relaciones. Y necesitará una competencia elevada en ALFIN, como paradigma educativo de primer orden. (Pinto, Sales y Osorio, 2009)

La innovación desde la biblioteca universitaria/CRAI debe estar enfocada esencialmente al desarrollo de servicios y estrategias que faciliten lograr un cambio en la manera en que los profesores y los estudiantes se relacionan con la información y el conocimiento. Este pensar de forma distinta constituye la base para lograr los siguientes cambios:

- Cambio en los objetivos trazados para el cumplimiento de la misión.

- Cambio en la proyección de los servicios informativos.

- Cambio en los procesos que soportan el desarrollo de la actividad de información en la universidad.

- Cambio en las relaciones que establece la biblioteca con el resto de los departamentos de la universidad y con sus usuarios.

- Cambio en los procesos formativos que se desarrollan desde la biblioteca.

Este nuevo escenario constituye un reto para los profesionales que trabajan en las bibliotecas universitarias y una premisa indispensable para 
abordar los servicios de información con un enfoque hacia la gestión de calidad total, centrada hoy más que nunca en la satisfacción del usuario y en la mejora continua.

\section{Metodología}

El estudio empírico-descriptivo que se presenta, es el resultado del análisis cualitativo de un focus group realizado en marzo de 2009, en el que participaron activamente 26 informantes iberoamericanos del Programa de Doctorado de Ciencias de la Documentación, que la Universidad de Granada imparte en la Universidad de la Habana. De ellos, 16 proceden de la titulación de biblioteconomía y ciencias de la documentación y el resto (10), de titulaciones tan diversas como Matemáticas, Letras, Comunicación, Educación, Ingeniería y Medicina. Trabajan como profesores en instituciones universitarias, bibliotecarios, documentalistas e informáticos en bibliotecas universitarias en los siguientes países: Argentina, Colombia, Cuba, Ecuador, Méjico y Venezuela.

La siguiente tabla contiene algunos datos sobre su formación inicial y el rol que desempeñan dentro de los entornos profesionales y universitarios.

\subsection{Focus group}

El origen del focus group se remonta a las llamadas dinámicas de grupo (Merton, 1956) y se define como una entrevista grupal, moderada por el docente, que se caracteriza por la interacción de los participantes. Recoge una serie de debates cuidadosamente planificados para obtener la percepción sobre un determinado tema de interés, en un ambiente adecuado (Krueger y Casey, 2009). Y se utiliza para recuperar información cualitativa, mediante la observación de los participantes y las entrevistas individuales (Morgan, 1997) y difiere de los métodos cuantitativos porque los datos no pueden ser usados a gran escala.

En nuestra práctica profesional es una técnica muy útil, sobre todo cuando el grupo de informantes que participa tiene una vasta experiencia y está involucrado fuertemente en el tema que se analiza, ya que permite recoger criterios que en muchas ocasiones suele coincidir con los resultados que se obtienen al utilizar técnicas que involucran una muestra mayor, sólo que en este caso se tiene la ventaja de poder observar los motivos y estados emocionales que manifiestan los participantes al expresar sus criterios.

\begin{tabular}{|c|c|c|}
\hline Titulación & Profesión actual & País \\
\hline $\begin{array}{l}\text { Bibliotecología/Ciencia de } \\
\text { la Información }\end{array}$ & Bibliotecaria & Cuba \\
\hline $\begin{array}{l}\text { Bibliotecología/Ciencia de } \\
\text { la Información }\end{array}$ & Bibliotecaria & Cuba \\
\hline $\begin{array}{l}\text { Bibliotecología/Ciencia de } \\
\text { la Información }\end{array}$ & Profesora & Cuba \\
\hline $\begin{array}{l}\text { Bibliotecología/Ciencia de } \\
\text { la Información }\end{array}$ & Profesor & Cuba \\
\hline $\begin{array}{l}\text { Bibliotecología/Ciencia de } \\
\text { la Información }\end{array}$ & Profesora & Argentina \\
\hline $\begin{array}{l}\text { Bibliotecología/Ciencia de } \\
\text { la Información }\end{array}$ & Profesor & Colombia \\
\hline $\begin{array}{l}\text { Bibliotecología/Ciencia de } \\
\text { la Información }\end{array}$ & Documentalista & Cuba \\
\hline $\begin{array}{l}\text { Bibliotecología/Ciencia de } \\
\text { la Información }\end{array}$ & Bibliotecaria & Cuba \\
\hline $\begin{array}{l}\text { Bibliotecología/Ciencia de } \\
\text { la Información }\end{array}$ & Documentalista & Cuba \\
\hline $\begin{array}{l}\text { Bibliotecología/Ciencia de } \\
\text { la Información }\end{array}$ & Documentalista & Cuba \\
\hline $\begin{array}{l}\text { Bibliotecología/Ciencia de } \\
\text { la Información }\end{array}$ & Profesor & Venezuela \\
\hline $\begin{array}{l}\text { Bibliotecología/Ciencia de } \\
\text { la Información }\end{array}$ & Profesora & Cuba \\
\hline $\begin{array}{l}\text { Bibliotecología/Ciencia de } \\
\text { la Información }\end{array}$ & Profesora & Cuba \\
\hline $\begin{array}{l}\text { Bibliotecología/Ciencia de } \\
\text { la Información }\end{array}$ & Bibliotecario & México \\
\hline $\begin{array}{l}\text { Bibliotecología/Ciencia de } \\
\text { la Información }\end{array}$ & Profesora & Cuba \\
\hline $\begin{array}{l}\text { Bibliotecología/Ciencia de } \\
\text { la Información }\end{array}$ & Documentalista & Cuba \\
\hline Computación & Informático & Ecuador \\
\hline Comunicación Social & Investigador & Colombia \\
\hline Educación & Bibliotecaria & Cuba \\
\hline Estomatología & Profesora & Cuba \\
\hline Farmacia & Documentalista & Cuba \\
\hline Ingeniero Agrónomo & Documentalista & Cuba \\
\hline Ingeniero Agrónomo & $\begin{array}{l}\text { Coordinador } \\
\text { Biblioteca }\end{array}$ & México \\
\hline Ingeniero Mecánico & Profesor & Cuba \\
\hline Letras & Documentalista & Cuba \\
\hline Matemática & Documentalista & Cuba \\
\hline
\end{tabular}

Tabla I. Datos de los profesionales que participaron en el estudio

Los objetivos del uso del focus group durante un proceso formativo son los siguientes:

1. Obtener información cualitativa de los participantes sobre determinados temas.

2. Explorar las necesidades de aprendizaje que tienen los participantes sobre los temas debatidos.

3. Percibir las expectativas de los participantes sobre la información que van a recibir en el curso sobre los temas tratados. 


\subsection{Descripción del focus group aplicado}

El grupo de enfoque fue estructurado de forma tal que facilitara la reflexión individual de los informantes y la interacción entre ellos mismos en el momento del debate. Las opiniones fueron transcritas de forma textual en formato digital, respetando cada detalle y manteniendo en el anonimato la identidad del informante.

El instrumento utilizado para la recogida de los datos fue pilotado previamente por tres profesionales, un docente y dos bibliotecarios, para evaluar si los datos recogidos y la redacción de las preguntas eran adecuadas para cumplir los objetivos de la investigación. El pilotaje fue favorable. Se elaboró la guía de entrevista y se recogió en una plantilla en soporte digital la información que cada uno de los informantes ofreció en su intervención. Se trabajaron con estos cinco ítems:

1. ¿Qué opinión te merece el proyecto de caminar hacia una universidad alfabetizada en información y qué papel han de jugar las bibliotecas en este contexto?

2. ¿Has participado en algún programa de innovación curricular en la biblioteca? En caso afirmativo, describelo, por favor. Indícanos si han colaborado docentes y estudiantes de algunas materias.

3. ¿Colaboras como profesional en el Campus Virtual de tu Universidad? Indícanos si desarrollas contenidos educativos.

4. En tú opinión, y según tú experiencia profesional, ¿qué beneficio supone para un estudiante estar alfabetizado en información?

5. ¿Cómo entrelazarías estos conceptos claves en la biblioteca del siglo XXI: evaluación, multialfabetismos, innovación y creatividad? Analiza sus interconexiones.

La sesión del grupo focal se realizó en abril del año 2009, como conclusión de un curso de doctorado sobre "Evaluación de la calidad en bibliotecas universitarias y servicios de información en el siglo XXI". Duró una hora y media y fue guiada por dos profesionales: uno, que actuaba de moderador, y otro, que transcribía la información.

Los debates se caracterizaron por un análisis profundo de los temas, vinculado sobre todo a la experiencia profesional de los informantes y a un conocimiento de los preceptos teóricos que definen los conceptos de alfabetización informacional, innovación y calidad y su relación con el presente y futuro de las bibliotecas universitarias.

\section{Resultados}

A continuación se manifiestan las principales ideas, conceptos y temas que fueron expuestos por los informantes (bibliotecarios y docentes) y que constituyen los patrones para este análisis. En el transcurso de la exploración se muestran conclusiones parciales que las autoras consideran oportuno destacar al final de cada ítem.

4.1. ¿Qué opinión te merece el proyecto de caminar hacia una universidad alfabetizada en información y qué papel han de jugar las bibliotecas en este contexto?

Sobre este aspecto, los informantes apuntaron los siguientes aspectos:

- La alfabetización informacional es vital en el trabajo de la biblioteca universitaria para el desarrollo de competencias informacionales y digitales en los estudiantes.

- La alfabetización informacional es importante para los docentes, porque ayuda a mejorar su rendimiento académico (especialmente, publicaciones).

- Los docentes deben entender la importancia que tiene estar entrenados en el manejo de la competencia informacional para el ejercicio de su actividad docente e investigadora. Además, deben transferir junto a bibliotecarios estas habilidades en su práctica docente para lograr que los estudiantes las integren en su proceso de aprendizaje.

- La alfabetización informacional ha de estar incluida de manera formal en el currículo de los diferentes títulos universitarios.

- La alfabetización informacional constituye un importante eje conductor para el aprendizaje del uso de las tecnologías de la información y la comunicación.

En general, pues, se evidencia que existe un consenso sobre la importancia de la alfabetización informacional para la formación de profesionales capaces de enfrentar los cambios que de forma continua se dan en la sociedad. Se reconoce la necesidad de que los bibliotecarios y docentes cooperan de forma estratégica y dominen las competencias informacionales que después han de enseñar a los estudiantes. La literatura consultada apoya estos planteamientos:

Snavely (2001) habla de la necesidad de lograr formas de cooperación entre bibliotecarios y docentes para que se oferten vías didácticas de calidad en ALFIN para el colectivo de estudiantes universitarios, teniendo muy en cuenta que la alfabetización informacional es una compe- 
tencia transversal esencial en la formación de todo profesional.

Para Okerson, (2004), la cooperación entre personal bibliotecario y docentes expertos en estudios de información es un elemento clave; y poner en marcha alianzas entre los departamentos y la biblioteca es una vía para promover el servicio socioeducativo que toda biblioteca debe proporcionar (Langley, Gray y Vaughan, 2006). Por tanto, es vital para el desarrollo de la alfabetización informacional la colaboración entre bibliotecarios, profesores y estudiantes (Sundin, Limberg y Lundh, 2008)

Otro tema presente en muchos escenarios ha sido la necesidad de integrar la formación en alfabetización informacional en toda propuesta curricular (Owusu-Ansah, 2004). En este sentido, podemos hacer uso de los elementos que a juicio de Webber y Johnston (2006) forman parte de una Universidad Alfabetizada:

1. Adecuada gestión de Alfin: estrategia, recursos, política e infraestructura.

2. Investigación alfabetizada en información.

3. Estudiantes y graduados alfabetizados en información.

4. Plan de estudios alfabetizado en información: reconocimiento de la Alfin como tema de estudio y como algo que atañe a la docencia, al aprendizaje y a la evaluación y calificación.

5. Formación del personal en Alfin.

6. Bibliotecarios alfabetizados en información.

Como se puede apreciar estas ideas complementan la necesidad expresa que han sentido los informantes de promocionar y desarrollar el proceso de alfabetización informacional desde la biblioteca universitaria, en coordinación con el resto de los integrantes de la institución académica.

4.2. ¿Has participado en algún programa de innovación curricular en la biblioteca? En caso afirmativo, descríbelo, por favor. Indícanos si han colaborado docentes y estudiantes de algunas materias.

Este interrogante sugirió un debate con una importante participación de los informantes, cuyas opiniones se pueden resumir de la siguiente forma:

- La biblioteca se tiene que integrar en los espacios docentes, y ha de fomentar la interacción entre bibliotecarios y docentes para incluir la alfabetización informacional en la educación superior (maestrías, grados, doctorados...).

- Programas para la formación en alfabetización informacional en la especialidad de biblioteconomía y ciencias de la información.

- Trabajo conjunto de bibliotecarios y docentes para desarrollar un modelo curricular de formación por competencias.

- Formación de bibliotecarios en ALFIN a través de cursos e-learning.

- Formación ALFIN en la universidad por niveles.

El análisis de las respuestas dadas por el colectivo profesional evidencia que aunque se han comenzado a dar pasos para la inclusión de contenidos relacionados con las competencias informacionales en la formación profesional, estos están encaminados principalmente al postgrado, donde existen resultados concretos en maestrías y doctorados.

No sucede así en el grado, donde queda mucho por hacer, ya que el proceso de apoderamiento sobre el tema es lento y no todas las universidades tienen trazadas estrategias para en plazos inmediatos puedan atender la formación de competencias informacionales en las diferentes disciplinas. Evidentemente la biblioteca universitaria hoy no está totalmente integrada en los procesos de innovación curricular donde deberían jugar un rol importante.

Autores destacados en el tema enfatizan tanto la necesidad de integrar la ALFIN en todo diseño curricular (Owusu-Ansah, 2004) como la de considerarla como disciplina, entendida como la adopción de un comportamiento informacional apropiado (Johnston y Webber, 2005).

La innovación pedagógica incide en la misión que cumplen las bibliotecas, ya que sus servicios y productos informativos deben ser lo suficientemente abiertos y dinámicos para adaptarse a los cambios educativos y tecnológicos que se producen en el interior de la universidad y a la vez lograr que se reconozca la necesidad de que los profesionales de la información participen junto a los profesores en el perfeccionamiento de los contenidos curriculares y la incorporación de la alfabetización informacional como materia obligatoria en el ciclo de aprendizaje.

Coincidimos con Bruce (1996) cuando afirma que la alfabetización informacional ha de consolidarse como herramienta formativa que capacite a los usuarios a la hora de desarrollar procesos informacionales. 
4.3. ¿Colaboras como profesional en el Campus Virtual de tu Universidad? Indícanos si desarrollas contenidos educativos

Las respuestas emitidas se refirieron a diferentes aspectos que bordean la problemática del Campus Virtual sin entrar en la esencia de la pregunta, lo cual puede indicar que la interrelación que se establece entre los profesionales de la información y el Campus Virtual de la Universidad no es ni todo lo suficiente, ni todo lo sistemático que debería ser para lograr un conocimiento profundo de este tema.

\section{Destacamos las siguientes aportaciones:}

- Desarrollo de sitios Web de las universidades con contenidos educativos para profesores y alumnos.

- Desarrollo de Bibliotecas Virtuales y Universidades Virtuales.

- Desarrollo de la filosofía de la Web 2.0 en bibliotecas universitarias.

- Uso de Moodle como plataforma para el aprendizaje on line.

- Desarrollo del Campus Virtual en las universidades.

La mayoría de los informantes interactúan con estudiantes universitarios en su formación postgraduada y reconocen que el uso de los espacios virtuales de aprendizaje se ha convertido en un elemento esencial para el desarrollo de las universidades y de sus bibliotecas.

Todos se muestran de acuerdo en la necesidad de evolucionar hacia modelos de bibliotecas mixtas, que combinen lo convencional con lo digital, como condición necesaria para no quedar fuera de los requerimientos que en materia de información nos exige el siglo XXI. Aunque, plantean que no siempre se encuentran creadas todas las condiciones para que los bibliotecarios colaboren en el campus virtual y aporten los contenidos suficientes para el desarrollo de competencias informacionales, ya sea por falta de preparación del bibliotecario o por limitaciones tecnológicas.

Pero, los bibliotecarios necesitan conocer mejor el modo en el que los usuarios se comportan actualmente en el entorno de una biblioteca virtual y cómo usan su costoso contenido (British Library y JISC, 2008). Además, los bibliotecarios que colaboren como docentes deberán saber crear entornos educativos significativos y programas de formación perdurables que sirvan para colmar las necesidades actuales y futuras de una persona como estudiante y como aprendiz a lo largo de la vida (ACRL, 2005).
En este sentido, las bibliotecas deberán formar nuevos especialistas con destrezas en áreas como producción de contenido digital, nuevos formatos metadatos, desarrollo de software y administración de sistemas, administración de proyectos, XML y otras destrezas técnicas. Simultáneamente, deberán comprender e interiorizar cómo los cambios en las prácticas de la comunicación académica afectan a la organización bibliotecaria en general (Rosenblum, 2008).

\subsection{En tú opinión, y según la experiencia} profesional, ¿qué beneficio supone para un estudiante estar alfabetizado en información?

Las respuestas a este interrogante tuvieron su base en el conocimiento previo que cada informante tenía sobre el concepto de alfabetización informacional y abordaron los siguientes parámetros de desarrollo de los estudiantes, resaltando estos beneficios:

- Supone estar preparados para la búsqueda, análisis, evaluación y uso ético de la información.

- Conlleva usar de manera adecuada las TiCs.

- Encajar la formación en el paradigma de aprender a aprender.

- Favorece el desarrollo del pensamiento crítico.

- Contribuye al proceso de gestión del conocimiento.

- Aprender a ser autónomos, actuar con independencia y tomar decisiones basadas en la información.

En general, se observa que los informantes reconocen que la introducción de la formación en ALFIN en la universidad de hoy, ya sea a partir de la inserción de los contenidos en los currículos o de la formación desde la propia biblioteca, se convierte en la piedra angular para formar individuos autónomos, capaces no solo de usar la información, sino de convertirla mediante el análisis y la reflexión en un nuevo conocimiento y así desarrollar una espiral donde de forma sistémica se vinculen los siguientes aspectos de esta ecuación:

Información - Conocimiento - Individuo Universidad - Sociedad.

Resulta importante constatar que hay un reconocimiento explicito y consciente de la relación entre la gestión del conocimiento que se debe producir en los entornos académicos y la presencia de los procesos de alfabetización informacional. Como señala Gómez, ALFIN debe ser un servicio básico a prestar a los usuarios, tanto de 
modo autónomo como en programas docentes más amplios y en colaboración con ellos (2000).

En el ámbito de la educación superior son varias las instituciones que han dedicado esfuerzo a elaborar modelos teóricos para la enseñanza de la alfabetización informacional (ACRL/ALA, 2000; SCONUL, 1999; ANZIL/CAUL, 2004). En la base de los modelos se describen las habilidades básicas que deben desarrollar los estudiantes para insertarse en la sociedad de la información. Según estas normas podemos concluir que los beneficios que obtienen los estudiantes al ser alfabetizados informacionalmente redundan en:

1. Ser capaz de determinar la naturaleza y nivel de información que necesita.

2. Evaluar la información requerida de manera eficaz y eficiente.

3. Incorporar la información seleccionada a su propia base de conocimientos y a su sistema de valores.

4. Utilizar la información eficazmente para cumplir un propósito específico.

5. Comprender muchos de los problemas y cuestiones económicas, legales y sociales que rodean al uso de la información, y accede y utiliza la información de forma ética y legal.

4.5. ¿Como entrelazarías estos conceptos claves en la biblioteca del siglo XXI: evaluación, multialfabetismos, innovación y creatividad?

Analiza sus interconexiones.

A este interrogante respondieron la mayoría de los informantes y resulta interesante el análisis de las respuestas dadas por el grupo de profesionales, por su enfoque holistico-sistémico:

- Desde la biblioteca se deben evaluar los procesos y actividades y visualizar la misión de la institución; cual es el perfil del egresado y cómo contribuye la biblioteca a este objetivo. Independientemente de que en el perfil del egresado esté el desarrollo de competencias, la biblioteca debe velar por su desarrollo.

- La evaluación para la mejora es un proceso continuo que va a definir las áreas donde hay puntos débiles y por tanto, hay que implementar programas de ALFIN.

- Todos estos conceptos están interrelacionados y a la vez interconectados en un proceso cíclico de flujo de información. Se crean contenidos para un programa ALFIN y a la vez esos contenidos derivan en resultados que implican una mejora en la calidad de los servicios y productos de la biblioteca.

- Es necesario desarrollar políticas nacionales de información que contemplen el desarrollo de la multialfabetización, la innovación y la evaluación en las bibliotecas, no solo a nivel de las universidades sino de todo el sistema de información.

\begin{tabular}{|c|c|}
\hline Pregunta & Conceptos emergentes \\
\hline $\begin{array}{l}\text { 1. ¿Que opinión te merece el } \\
\text { proyecto de caminar hacia una } \\
\text { universidad alfabetizada en } \\
\text { información y que papel han de } \\
\text { jugar las bibliotecas en este } \\
\text { contexto? }\end{array}$ & $\begin{array}{l}\text { Proactividad } \\
\text { Interdisciplinariedad y } \\
\text { convergencia de } \\
\text { servicios } \\
\text { Alianzas estratégicas }\end{array}$ \\
\hline $\begin{array}{l}\text { 2. ¿Has participado en algún } \\
\text { programa de innovación } \\
\text { curricular en la biblioteca? En } \\
\text { caso afirmativo, describelo, por } \\
\text { favor. Indícanos si han } \\
\text { colaborado docentes y } \\
\text { estudiantes de algunas materias }\end{array}$ & $\begin{array}{l}\text { Formación } \\
\text { contextualizada } \\
\text { Formación en cascada } \\
\text { Formación del personal } \\
\text { Integración curricular } \\
\text { Formación por } \\
\text { competencias }\end{array}$ \\
\hline $\begin{array}{l}\text { 3. ¿Colaboras como profesional } \\
\text { en el Campus Virtual de la } \\
\text { Universidad? Indícanos si } \\
\text { desarrollas contenidos } \\
\text { educativos. }\end{array}$ & $\begin{array}{l}\text { Aprendizaje e-learning. } \\
\text { Web } 2.0 \\
\text { Desarrollo de espacios } \\
\text { virtuales } \\
\text { Plataforma para el } \\
\text { aprendizaje }\end{array}$ \\
\hline $\begin{array}{l}\text { 4. En tu opinión, y según tu } \\
\text { experiencia profesional, ¿que } \\
\text { beneficio supone para un } \\
\text { estudiante estar alfabetizado en } \\
\text { información? Señala cual sería } \\
\text { a tu juicio la mejor práctica }\end{array}$ & $\begin{array}{l}\text { Autonomía } \\
\text { Pensamiento crítico } \\
\text { Aprender a aprender }\end{array}$ \\
\hline $\begin{array}{l}\text { 5. ¿Como entrelazarías estos } \\
\text { conceptos claves en la } \\
\text { biblioteca del siglo XXI: } \\
\text { evaluación, multialfabetismos, } \\
\text { innovación y creatividad? } \\
\text { Analiza sus interconexiones. }\end{array}$ & $\begin{array}{l}\text { Innovación } \\
\text { Evaluación } \\
\text { Políticas de } \\
\text { información } \\
\text { Gestión de calidad total }\end{array}$ \\
\hline
\end{tabular}

Tabla II. Tabla resumen

Es importante destacar cómo la gran mayoría de los informantes reflexionan en torno a la formulación de que evaluación, multialfabetismos, innovación y creatividad son conceptos que se complementan en el desarrollo de las bibliotecas. Dicho como trabalenguas sería así: La evaluación conlleva a la mejora de la calidad y al desarrollo de creatividad e innovación en las organizaciones. La innovación y la creatividad elevan la calidad de los servicios y productos que se ofrecen en las bibliotecas. La multialfabetización estimula la innovación y la creatividad y deriva hacia la mejora de la calidad de todos los procesos que se generan en la sociedad. Todos necesitan de todos para afrontar los desafíos de la sociedad de la información y el desarrollo de nuevos modelos de servicios integrados que tengan como misión, visión y meta 
al usuario, tanto en su faceta de consumidor como de prosumidor de información.

La comunidad de bibliotecarios y estudiosos han manifestado interés y a la vez preocupación por la falta de rigor y de métodos adecuados para la evaluación de la alfabetización informacional y la labor de la biblioteca en la educación superior (Walsh, 2009). En la literatura especializada se plantea que la evaluación del rendimiento de la biblioteca debe quedar definida y conformada por sus conexiones y sus contribuciones a las metas y a los resultados educativos previstos para la institución en su conjunto. Así mismo se insiste en que la evaluación debe reflejar la relación de la biblioteca con las funciones de enseñanza y aprendizaje de la institución (Wolff, 1995, Hernon and Whitman, 2001).

Las bibliotecas que han pasado por un proceso de certificación de calidad (EFQM, 2003; UNEEN ISO, 2008) están mucho más preparadas para afrontar los procesos de innovación, de creatividad y de alfabetización informacional en el proceso de enseñanza-aprendizaje.

\section{Conclusiones}

Teniendo en consideración la formación profesional, procedencia y experiencia de la muestra, además de la activa participación de los miembros del grupo en el análisis exploratorio realizado durante el focus group, se considera que los elementos aportados son representativos sobre este tema, destacándose como esenciales las siguientes conclusiones:

1. La biblioteca universitaria/CRAI en colaboración con los académicos, tiene que participar en la definición e implementación de los Programas de Alfabetización Informacional dentro de la universidad. De una actitud pasiva debe pasar a una actitud proactiva que logre dinamizar el proceso de gestión de información y conocimiento que de forma constante se produce en la universidad.

2. No existe una suficiente vinculación de los bibliotecarios en los procesos de innovación curricular de la universidad, ni una apuesta colectiva e integrada de lo que implica y supone la formación por competencias de los distintos agentes de la comunidad universitaria (bibliotecarios, docentes y estudiantes)

3. Se reconoce la importancia del Campus Virtual como soporte al proceso de aprendizaje basado en un nuevo modelo pedagógico que apuesta por un estudiante autónomo, que utilice intensamente las tecnologías de información y que construya su propio proceso de auto-aprendizaje.

4. La alfabetización informacional y el desarrollo de competencias informacionales estarán entre las principales prioridades de los profesionales de la información por los beneficios que supone para aquellos que logran aprender a utilizar la información desde una perspectiva holistica e integradora.

5. Los conceptos y procesos de evaluación, innovación y creatividad se identifican como las aristas que garantizan la calidad de los procesos de gestión de información y del conocimiento que deben desarrollarse en las bibliotecas universitarias, como condición indispensable para formar profesionales competentes en el manejo y uso adecuado de la información.

\section{Bibliografía}

ACRL/ALA. (2000). Normas sobre aptitudes para el acceso y uso de la información en la Educación Superior. // Boletín de la Asociación Andaluza de Bibliotecarios. 15 (60). http://www.aab.es/51n60a6.pdf (2009-09-10).

ACRL-IS/RSC. (2005). Agenda para la investigación en instrucción bibliográfica y alfabetización informacional (ALFIN). // Anales de la Documentación. 8 (2005) 275283. http://revistas.um.es/analesdoc/article/viewFile/ 3241/3201 (2009-10-15).

ANZIL/CAUL. (2004). Normas de Alfabetización Informacional. http://www.aab.es/pdfs/gtbunormas08.pdf (2009-0320).

Area, M. (2004). De la Biblioteca Universitaria al centro de recursos para el aprendizaje e investigación. Informe final. Madrid: Dirección General de Universidades.

Beagle, D. (1999). Conceptualizing and Information Commons. // Journal of Academic Librarianship. 25:2 (1999) 82-89.

Buttlar, L; Tipton, M. (1992). Library Use and Staff Training in Curriculum Materials Centres. // The Journal of Academic Librarianship. 17:6 (1992) 370-74.

British Library; JISC. (2008). Informe ClBER.Comportamiento Informacional del investigador del futuro. // Anales de la documentación. 11 (2008) 235258

Bruce, C. (1996). Information Literacy: How do University Educators Understand this Phenomenon? // Booker, D. (ed.), Learning for life: Information Literacy and the Autonomous Learner, Adelaida: University of South Australia, 78-86.

Bruce, H. (2009). Global Research Library 2020. Event Guide and Position Paper. http://www.grl2020.net/ (2009-09-20)

Cano, V. (1996).Information technology and the future of professional Library practice. // Geh, H. P.; Walckiers, M. (Eds.), Library networking in Europe. Londres: TFPL, 5165.

Carman, J. M. (2002). Blended Learning Desing: Five Key Ingredients. http://knowledgenet.com/pdf/Blended\%20 Learning\%20Desing_1028.PDF (2009-09-22). 
Cunningham, P.N. (2006). European Trend Chart on Innovation, Policy Review Workshop 10: "Innovation in Services", Workohop Input Report.

Dominguez, M. I. (2005). La biblioteca universitaria ante el nuevo modelo de aprendizaje: docentes y bibliotecarios, aprendamos juntos porque trabajamos juntos. // RED. Revista de Educación a Distancia, número monográfico II. http://www.um.es/ead/red/M4/ (2009-10-04).

European Foundation for Quality Management (2003). Modelo EFQM de excelencia. Bruselas: EFQM. http://www.efqm.org/uploadsintroducing_spanish.pdf (2009-10-10)

GarrisoN, D.R.; Kanuka, H. (2004). Blended Learning: Uncovering its Transformative Potential in Higher Education // The Internet and Higher Education. 7:2 (2004) 95-105.

Gómez, J. A. (2000). La Alfabetización informacional y la biblioteca universitaria, organización de programas para enseñar el uso de la información. // Gómez, J. A. (coord.) Estrategias y modelos para enseñar el uso de la información. http://eprints.rclis.org/archive/00004672/05/ EMPEUIcap4.pdf (2009-100-06).

Hernon, P.; Whitman, J. Delivering Satisfaction and Service Quality. Chicago: American Library Association, 2001.

Howells, J. (2000) Services and systems of innovation. // Andersen, B; Howells, J.; Hull, R.; Miles, I.; Roberts. J. (eds), Knowledge and Innovation in the New Service Economy, Cheltenham: Edward Elgar, 215-228

Johnston, B.; Webber, S. (2005). As we may think: Information literacy as a discipline for the information age. // Research Strategies. 20:3 (2005) 108-121

Kazmer, M. (2002). Distance Education students speak to the library: hers how you can help even more. // The Electronic Library. 20:5 (2002) 395-400.

Krueger, R.A.; Casey, M.A. (2009). A practical Guide for Applied Research. 4ta Ed. Thousand Oaks, CA: Sage Publications.

Langley, A.E.; Gray, E.; Vaughan, K.T. (2006). Building Bridges: Colaboration within and beyond the academic library. Oxford: Chandos.

Merton, R. K.; Kendall, P. L.; Fiske, M. (1956). The focused interview: A manual of problems and procedures. Glencoe, IL.: Free Press

Morgan, D. L. (1997). Focus Group. As Qualitative Research. Qualitative Research Methods.16:2.

Moscoso, P. (2003). La nueva misión de las bibliotecas universitarias ante el Espacio Europeo de la Enseñanza Superior. // Jornadas Rebiun: Los Centros para recursos del aprendizaje y la investigación docente. http://biblioteca.uam.es/paginas/palma.html. (2009-0902).

Okerson, A. (2004). La biblioteca digital: asteroides, la ley de Moore y la Star Alliance. // Anales de la Documentación 7 (2001) 263-273.

Owusu-Ansah, E.K. (2006). Knowledge Organization and Disseminatio, and Knowledge Navigation and the Library Meet in Higher Education. // The International Journal of Learning. 12:1 (2006) 1-8.

Oyston, E. ed. (2003). Centred on Learning: Academia Case Studies on Learning Centre Development. Londres: Ashgate.

Pinto, M.; Sales, D.; Martínez, P. (2009). El personal de la biblioteca universitaria y la alfabetización informacional: de la auto percepción a las realidades y retos formativos. // Revista Española de Documentación Científica. 32:1 (2009) 60-80.

Pinto, M. (2008). Alfabetización informacional y e-learning. Diseño de tutoriales y cursos on line. // Gómez, J. A.; Calderon, A. (Eds), Brecha digital y nuevas alfabetiza- ciones. El papel de las bibliotecas. Madrid: Universidad Complutense. /OLibro.pdf (2009-04-12).

Pinto, M.; Balagué N.; Anglada, L. (2007). Evaluación y calidad en las bibliotecas universitarias: experiencias españolas entre 1994-2006. // Revista Española de documentación científica. 30:3 (2007) 364-383.

Pinto, M.; Sales, D.; Osorio, P. (2008). Biblioteca Universitaria, CRAl y alfabetización informacional. Gijón, Trea, 2008.

Rosenblum, B. (2008). Desarrollo de nuevas destrezas y competencias para apoyar la producción académica digital y la comunicación académica, World Library and Information Congress: 74 TH IFLA General Conferwence and Council. http://docs.google.com/gview?a $=v \& q=$ cache:js4sUXBYrRMJ:ifla.queenslibrary.org/IV/ifla 74/papers/150-Rosenblumtranses.pdf+\%29.+Desarrollo + de+nuevas+destrezas+y+competecias+para+apoyar+ la+producci\%C3\%B3n+acad\%C3\%A9mica+digital+y+la +comunicaci\%C3\%B3n+acad\%C3\%A9mica\&hl=es\&gl= es\&sig=AFQjCNELEhwvjpZRMxo62MXKrS4tkB3Sdw (2009-09-10).

Sánchez, B. (2007). Ciencia, investigación y cultura en la biblioteca universitaria actual. // Acimed. 15:1. http://bvs.sld.cu/revistas/aci/vol15_1_07/aci16107.htm (2009-10-06).

Sconul. (1999). Aptitudes para el acceso y uso de la información en la enseñanza superior: la postura de Sconul. Informe preparado por el grupo de trabajo Sconul sobre Aptitudes para el Acceso y Uso de la Información. http://www.aab.es/pdfs/baab62/62a4.pdf (2009-09-04).

Snavely, L. (2001). Information Literacy standards for higer education: and international perspective. // 67 IFLA council and General Conference, 16-25, agosto del 2001. Proceeding. The Hague, IFLA. http:// www.ifla.org//V/ifla67/papers/073-126e.pdf (2009-09-30).

Sundin, O.; Limberg, L.; Lundh, A. (2008). Constructing librariansns' information literacy expertise in the domain of nursing. // Journal of Librarianship Information Science. 40:1 (2008) 21-30.

UNE-EN ISO 9001(2008). Sistemas de gestión de la calidad. Requisitos. Madrid: AENOR.

University Grants Commitee (1976). Capital provision for university Libraries (Informe Atkinson) Londres: HMSO.

Walsh, A. (2009). Information literacy assessment: Where do we start. // Journal of Librarianship Information Science. 41:1 (2009) 19-28.

Webber, S.; Johnston, B. (2006). Working towards the Information Literate University. // Walton. G.; Pope, A. (Eds.), Information Literacy: Recognising the need). Oxford: Chandos. 42-53.

Wolff, A. (1995). Using the accreditation process to transform the mission of the library. // New directions for Higher Education. 90 (1995) 79.

Zabalza, M. A. (2003). Innovación en la enseñanza universitaria. // Contextos educativos: Revista de educación. 67, 113-136. http://dialnet.unirioja.es/servlet/oaiart?codigo $=1049473(2009-09-30)$.

Recibido: 06-07-2010. Aceptado: 06-07-2010. 
